\title{
Impacto de la gestión y políticas públicas en el desarrollo de proyectos de inversión en el AA.HH. olof palme del distrito de Chilca, Cañete 2021
}

Santiago Linder Rubiños Jiménez srubinos@ucvvirtual.edu.pe

Miguel Constantino Nieves Barreto cmnievesb@unac.edu.pe

Yesmi Katia Ortega Rojas

ykortegar@unac.edu.pe

Fernando Emilio Escudero Vílchez fescudero@silfersystem.com

Silvia Liliana Salazar Llerena ssalazar@silfersystem.com

Doctor en Gestión Pública y Gobernabilidad en la Universidad César Vallejo

Lima - Perú

\section{RESUMEN}

OBJETIVO: Determinar el impacto de la gestión y políticas públicas en el desarrollo de proyectos de inversión en el A.A.H.H. Olof Palme del distrito de Chilca, Cañete, 2021. METODOLOGÍA: Bajo un estudio cuantitativo básico aplicado de nivel correlacional, y un diseño no experimental transeccional descriptiva, la técnica empleada fue la encuesta como instrumento de recolección de datos el cuestionario. Asimismo, la población en estudio es de 85 funcionarios de la municipalidad y 35 de ellos fueron la muestra. CONCLUSIÓN Y RESULTADOS: El objetivo general, determinar el impacto de la gestión y políticas públicas en el desarrollo de proyectos de inversión, es aceptada con un nivel de significancia de 0,000 y un Chi cuadrado de 358,741. De tal manera que, se observa el valor del coeficiente Nagelkerke de $98,1 \%$, lo que también indica que es aceptada la hipótesis planteada por el investigador, por lo tanto, se valida el nexo entre las variables.

Palabras clave: políticas públicas; gestión y proyectos de inversión. 


\title{
Impact of management and public policies on the development of investment projects in the olof palme housing estate in the district of Chilca, Cañete 2021
}

\begin{abstract}
OBJECTIVE: To study the incidence of Distance Education as a Public Policy in the Achievement of Competences in Higher Education students. METHODOLOGY: Under a basic quantitative study applied at a correlational level, and a descriptive transectional non-experimental design, the technique used was the survey and the data collection instrument was the questionnaire. Likewise, the population under study is 115 students of the last year of studies of the Academic Area of Computing and Informatics and teachers of the IESTP. "Manuel Arévalo Cáceres" -Los Olivos, Lima, Peru. CONCLUSION AND RESULTS: The general objective, public policies in distance education are related to the achievement of competencies in the subjects of study, is accepted with a significance level of 0.000 and a Pearson's Chi-square of 15.369. In collaboration with Spearman's Rho correlation, the figure of 0.413 was obtained, whose value is of moderate level, therefore, the nexus between the variables is validated.
\end{abstract}

Key words: public policies; distance education and competencies.

Artículo recibido: 30 noviembre. 2021 Aceptado para publicación: 29 diciembre 2021 Correspondencia: srubinos@ucvvirtual.edu.pe Conflictos de Interés: Ninguna que declarar 


\section{INTRODUCCIÓN}

En la actualidad, se ha podido percibir que es muy importante que exista una buena gestión para que se puedan desarrollar los proyectos de manera eficiente, sin embargo, debido a la pandemia del COVID-19, hubo cambios a los cuales debieron adaptarse para seguir en el cumplimiento de sus objetivos, ya que ha ocasionado algunos problemas para los cuales los países no estaban listos de afrontar (Avalos \& Brenes, 2021). Muchos se han visto forzados a cambiar y atraer nuevos métodos para la realización de sus actividades, uno de ellos ha sido la gestión de proyectos, debido a que este ha tenido que realizarse abarcando una serie de herramientas que permitan brindar la eficacia que se necesita en todo aspecto (Dauzón \& Izquierdo, 2020).

Soto (2021) hace referencia a que la ejecución de proyectos de inversión pública toma en consideración muchas herramientas para que se cumplan los objetivos con el fin de prestar un mejor servicio a la calidad de vida de los ciudadanos lo cual incliye también a las obras públicas, ya que con el paso de los años, se han imposibilitado la optimización de la eficiencia de estos proyectos cuando se debe superar dichas dificultades para satisfacer las necesidades básicas de los ciudadanos. Sin embargo, las políticas públicas son los que también intervienen en las decisiones gubernamentales, ya que ellos lo ven desde la perspectiva del Estado a través de las funciones y fines de este, la acción estatal incluye a aquellos programas, proyectos y planes para poder llegar a todos los distritos que requieren de personas que se encuentren enfocadas en el desarrollo en los ámbitos como salud, educación, economía, entre otros (Gutiérrez et.al, 2017).

Para Morveli (2021), la gestión pública y su influencia en el gobierno peruano ha llegado a diferentes niveles ya que se desarrollaron enfoques de acuerdo a lo que requería la población en diversos ámbitos, lo cual se optimiza a través de los objetivos, la gestión por procesos así como la de calidad y la gestión estratégica; todos ellos van orientados a alcanzar la satisfacción del ciudadano de manera progresiva. Jiang (2021) considera que el capital humano desempeña un gran rol para el crecimiento económico, de tal manera que sus actividades pueden servir como utilidad para la maximización de la acumulación de capital en cuanto a la salud, lo que brinda un gran aporte a los decisiones que se pueden tomar para mostrar que el capital humano es el adecuado, aparte que se ciñen a los modelos de desarrollo en forma, después de analizar todos ellos, se emplea el más adecuado para la sociedad. 
Ola-Awo et.al (2021) el desarrollo de proyectos se da en la práctica de la gestión de aquellos que se encuentran interesados, en los objetivos se tiene en cuenta los plazos, sobrecostes y disputas así como el analizar la falta de confianza entre los interesados; para la gestión de proyectos se requiere de la construcción, se determinó que son importantes siete factores críticos como la participación, el contar con líderes de proyectos, la formulación de la misión clara del proyecto, el manejo de una estrategia empresarial, el apoyo en la dirección así como la difusión de información ya que esta permite que se establezcan relaciones con el fin de alcanzar el éxito y el rendimiento del proyecto.

Zavaleta \& Cruz (2020) señalan que en relación con la evolución de la productividad que se encuentra orientado hacia la demanda con el fin de tener crecimiento en cuanto a la productividad, la demanda se puede visualizar en la forma de las ventas, en la capacidad del personal en la cual se puso en marcha la economía a través de escalas dinámicas y estáticas que son aquellas que reflejan la reducción entre la relación capital y producto; la demanda efectiva se llega a relacionar directamente con la expansión del gasto público, un claro ejemplo es la importación. Asimismo, para poder analizar la contribución de la inversión pública se toma en cuenta una serie de acontecimientos y cambios que ocurren en la empresa, así como el manejo de las empresas y negocios que permiten que las actividades se desarrollen de mejor manera aprovechando los recursos que los ayudan a crecer, tales como las inversiones públicas que se relaciona con lo financiero y económico, el que genera resultados óptimos con beneficios tanto para el comercio como para el personal, de cierto modo, las empresas deben estar preparadas para los cambios y la competencia que se genera con la creación de nuevas empresas, teniendo ello en consideración, esta se puede colocar en un buen lugar dentro del mercado comercial (Huacchillo et.al, 2020).

La gestión pública se hace cargo de la gestión de proyectos, la cual permite la generación para poder integrar factores sobre el gobierno electrónico y la gestión de la misma, con el fin de obtener buenos resultados en el Estado y en el sector privado, se consolidan estrategias que permiten la ejecución de estos proyectos para tener una ventaja competitiva en el desempeño de las empresas, a pesar de las dificultades, la implementación de políticas públicas aportan en el entorno de los proyectos y brinda la posibilidad de ampliar los servicios que son prestados a través de inversiones de nuevas acciones que favorecen a los ciudadanos (da Assunção \& Rabechini, 2020). 
La investigación surge por la necesidad de determinar el impacto de la gestión y políticas públicas en el desarrollo de proyectos de inversión en el A.A.H.H. Olof Palme del distrito de Chilca, Cañete, 2021; lo que se relaciona con investigar cuales son las herramientas que se emplean para el desarrollo de los proyectos de inversión así como el manejo de la gestión pública, en el cual se implementan a través de proyectos, programas, personal capacitado, con el fin de brindar lo mejor a los ciudadanos y que estos se sientan satisfechos (Holst Volden, 2018).

\section{Problema General}

- ¿Cuál es el impacto de la gestión y políticas públicas en el desarrollo de proyectos de inversión en el A.A.H.H. Olof Palme del distrito de Chilca, Cañete, ¿2021?

\section{Problemas específicos}

- ¿Cuál es el impacto de la gestión y políticas públicas en el rol de los gobiernos regionales y locales para el desarrollo de proyectos de inversión en el A.A.H.H. Olof Palme del distrito de Chilca, Cañete, 2021?

- ¿Cuál es el impacto de la gestión y políticas públicas en la inversión y crecimiento económico de los proyectos de inversión en el A.A.H.H. Olof Palme del distrito de Chilca, Cañete, 2021?

- ¿Cuál es el impacto de la gestión y políticas públicas en los lineamientos para la eficacia los proyectos de inversión en el A.A.H.H. Olof Palme del distrito de Chilca, Cañete, 2021 ?

\section{Objetivo General}

- Determinar el impacto de la gestión y políticas públicas en el desarrollo de proyectos de inversión en el A.A.H.H. Olof Palme del distrito de Chilca, Cañete, 2021.

\section{Objetivos específicos}

- Determinar el impacto de la gestión y políticas públicas en el rol de los gobiernos regionales y locales para el desarrollo de proyectos de inversión en el A.A.H.H. Olof Palme del distrito de Chilca, Cañete, 2021.

- Determinar el impacto de la gestión y políticas públicas en la inversión y crecimiento económico de los proyectos de inversión en el A.A.H.H. Olof Palme del distrito de Chilca, Cañete, 2021. 
- Determinar el impacto de la gestión y políticas públicas en los lineamientos para la eficacia en los proyectos de inversión en el A.A.H.H. Olof Palme del distrito de Chilca, Cañete, 2021.

\section{Hipótesis General}

- La gestión y políticas públicas impacta positivamente en el desarrollo de proyectos de inversión en el A.A.H.H. Olof Palme del distrito de Chilca, Cañete, 2021.

\section{Hipótesis específicas}

- La gestión y políticas públicas impacta positivamente en el rol de los gobiernos regionales y locales para el desarrollo de proyectos de inversión en el A.A.H.H. Olof Palme del distrito de Chilca, Cañete, 2021.

- La gestión y políticas públicas impacta positivamente en la inversión y crecimiento económico de los proyectos de inversión en el A.A.H.H. Olof Palme del distrito de Chilca, Cañete, 2021.

- La gestión y políticas públicas impacta positivamente en los lineamientos para la eficacia de los proyectos de inversión en el A.A.H.H. Olof Palme del distrito de Chilca, Cañete, 2021.

\section{Antecedentes}

Lakerveld et al. (2020) en su artículo "Advancing the evidence base for public policies impacting on dietary behavior in Europe: The Policy Evaluation Network promoting a multidisciplinary approach", tuvo como fin analizar las acciones llevadas a cabo por la Red de Evaluación de Políticas (REN), el cual analiza el contenido y la implementación de medidas públicas direccionadas a brindar una vida saludable en la población. Se realizó una revisión bibliográfica y un procesamiento de datos a fin de contar con información relevante de la problemática a estudiar; se concluyó que las políticas públicas implementadas por países en Europa han logrado resultados favorables en cuanto los comportamientos del estilo de vida de la población, logrando mejorar y permitiendo una mayor calidad de vida en estos.

De la misma manera, Fleury et al. (2018) en su investigación titulada "Public policies and the city: producing inclusing urban spaces" precisa estudiar acerca de los proyectos de inversión en torno a los espacios urbanos, analizando el dinamismo y los beneficios que genera para la población. Se indicó que los proyectos políticos se encuentran dirigidos a implementar ambientes urbanos, los cuales permitan que la ciudadanía ejerza su derecho 
de disfrute en la ciudad mas para ello se debe contar con inversión que permita ejecutar este tipo de proyectos.

Brunet (2019) en su artículo de investigación “Governance-as-practice for major public infrastructure projects: A case of multilevel project governing" planteó como principal objetivo llevar a cabo una conceptualización acerca de la gobernanza de proyectos, ello basándose en corrientes de desarrollo actuales; la investigación contó con un enfoque cualitativo y estudio un caso múltiple de infraestructura pública llevado a cabo en Quebec, Canadá. Se concluyó que los principales aportes que se han obtenido a partir de la gobernanza de proyectos son el desarrollo de un marco institucional que ha permitido el aumento de número de proyectos y la documentación y enseñanza sobre la gobernanza. También, Hong y Ryu (2019) en su artículo "Crowdfunding public projects: Collaborative governance for achieving citizen co-funding of public goods" precisó como objetivo general explorer acerca del potencial del crowdfunding con respecto cofinanciamiento de políticas y proyectos públicos. La investigación empleó una plataforma de crowdfunding coreana del año 2016, lo cual permitió analizar los proyectos con mayor transparencia que han logrado éxitos en la comunidad; se concluyó que una gobernanza colaborativa entre el sector público y privado permite lograr mayores proyectos de inversión en favor de la ciudadanía, siendo así que se determinó que el crowdfunding es potencialmente beneficioso.

Damascene y Andersson (2019) precisa en su artículo de investigación "The public value of E-Government - A literatura review" como principal objetivo analizar el rendimiento del gobierno electrónico en la actualidad y su relación con el valor público. Se realizó una revisión de la literatura acerca de la problemática estudiada, asimismo se empleó los criterios de tiempo de publicación, información relevante y el idioma. Se concluyó que el gobierno electrónico ha permitido en cuanto al sector público distintos beneficios, entre los cuales se pudo resaltar la mejora de los servicios públicos, esto a través del ofrecimiento a la ciudadanía de servicios de calidad, una mayor eficacia de la administración, un gobierno que plantea mayor inversión para políticas públicas, y mejoramiento de la capacidad del gobierno, una mayor transparencia y comunicación.

Wilson (2022) en su artículo "Public engagement and AI: A values analysis of national strategies" pretende analizar la influencia de la inteligencia artificial en la gestión pública mediante las estrategias, se empleó la revisión sistemática y análisis sobre 16 estrategias 
nacionales del año 2020 parte de la Organización para la Cooperación y el Desarrollo Económico (OCDE) que emplearon inteligencia artificial. Se concluyó que la participación en tanto a la gobernanza de la inteligencia artificial en los países es escasa, así como la comunicación con la ciudadanía sobre las políticas públicas tecnológicas que se pretenden plantear; se señaló que se debe promover la participación del sector público y privado con la intención de impulsar este tipo de proyectos y ofrecer a la ciudadanía soluciones a través de estas estrategias.

Por último, Arundel et al. (2019) en su artículo de investigación "Advancing innovation in the public sector: Aligning innovation measurement with policy goals" precisó como principal objetivo analizar las políticas que apoyan la innovación en el sector público. Se concluyó que el interés que debe existir con respecto a las políticas de innovación deben incluir la gobernanza y la gestión de innovación, en cuanto se pretenda lograr innovar el sector público en función a la globalización y uso de tecnologías de información y comunicación que se afrenta en la actualidad, asimismo precisa que se debe conocer los obstáculos que no permiten la aplicación de estas políticas, esto ya que no solo permitiría su solución sino también proveería de conocimiento para las diferentes áreas en torno a la administración pública.

\section{Teorías}

En cuanto a la dirección de proyectos públicos, se encuentran distintas perspectivas teóricas, tal como señalan (Wagner, Huemann, \& Radujkovic, 2021) la proyectificación de la empresa señala que existe una red de socios que se encuentran reconfigurando el marco de un proyecto y a la vez trabajan en otros proyectos secuenciales relacionados al que se encuentran reconfigurando, esto permite contar con una red de proyectos en un campo, además se precisa que la proyectificación genera efectos positivos en el área económica y laboral. La teoría institucional de la proyectificación señala que existen actores colectivos que trabajan de manera conjunta persiguiendo proyectos, estos trabajan de manera institucional y pretenden brindar estabilidad y significado a la vida social, asimismo resaltan que las asociaciones profesionales influyen en el comportamiento social y buscan ampliarse a los distintos sectores, ya sean públicos o privados, implementando y aplicando normativas, normas y estándares que viabilicen la gestión de proyectos. 
Asimismo, (Hansonn, 2020) precisa en torno a la teoría de las políticas, la cual permite conocer la función que sostiene el administrador en la adaptación de políticas, que se poseen cuatro fases, las cuales son definición y establecimiento de la problemática, formulación y adaptación de la política, implementación y finalmente evaluación, cuando se pretende implementar una política en una organización. La teoría se entorna en la fase de formulación y adaptación de políticas, ya que los directores o administradores empleando su conocimiento sobre la problemática al contar con la propuesta de política comienzan a estudiarla y formular planes, así como emplear recursos, para proyectar dicha política en el lugar que se pretende a llevar a cabo, esto para medir los costos, beneficios, entre otros, que lograría tras haberse ejecutado. En el sector público estos administradores al ser contratados por el Estado deben valorar y practicar la imparcialidad, objetividad y no politización de los proyectos que se pretenden ejecutar.

\section{ESTRATEGIAS METODOLÓGICAS O MATERIALES Y MÉTODOS}

\section{Tipo y Diseño de Investigación}

El presente estudio tuvo como objetivo general, determinar el impacto de la gestión y políticas públicas en el desarrollo de proyectos de inversión. Se tuvo una investigación cuantitativa básica, es decir se recopilará información para las bases teóricas de las respectivas variables, de tal manera que se ahonde en el tema. Aplicada y de nivel descriptivo-explicativo, ya que se intenta medir la relación entre ambas variables, sin que el investigador interfiera o controle alguna de ellas, de tal modo que en este estudio se busca establecer y evidenciar la relación entre las variables de estudio. Se utilizó el diseño de investigación no experimental transeccional descriptiva, debido a que no se buscó cambiar el contexto, sino estudiarlas en él.

\section{Población y Muestra}

- Como población se consideró a 85 funcionarios de la municipalidad de Chilca.

- Como muestra se seleccionó 35 funcionarios de la municipalidad de Chilca.

\section{Técnica e Instrumento de Recolección de Datos}

La técnica que fue empleada para llevar a cabo la investigación, es la encuesta en escala de Likert y como instrumento de recolección de datos el cuestionario, uno para cada variable, el cual será aplicado a la muestra en estudio. 


\section{Procedimientos}

La recolección de información se realiza en tres pasos, el primer paso se centra en obtener datos importantes acerca del centro en investigación y de los sujetos de estudio, asimismo, se obtuvo un permiso de datos e información general, de modo que estos datos sean confidenciales. El segundo paso se centra en el contraste de fuentes en base a información recolectada de otras investigaciones, nacionales e internacionales, esto para que el presente estudio pueda tener información variada y verídica. El tercer paso se centra en la realización de un cuestionario acerca de las variables, de tal manera que la muestra en estudio pueda resolver las dudas y brindar información de primera mano, es importante señalar que fue validado debidamente por los expertos en base a la prueba Binominal, seguidamente se analizará en una base de datos, mediante uso de tablas y gráficos.

\section{Método de Análisis de Datos}

Asimismo, se utilizó la escala de Likert y el formulario Google forma para que la información sea ordenada y precisa, y como instrumento de recolección el cuestionario. Del mismo modo, para las respuestas de los instrumentos se usó como tabulación el programa estadístico SPSS V26, con la ayuda del método estadístico descriptivo: El cual es un método que permite que la investigación pueda obtener tablas y gráficos con los datos recogidos. Por último, se hizo uso del método estadístico inferencial, esto con el fin de contrastar las hipótesis de investigación planteadas en base al Alfa de Cronbach.

\section{RESULTADOS Y DISCUSIÓN}

\section{A. Resultados}

A continuación, se describen los resultados a través de las tablas que contienen datos recopilados a partir de las encuestas aplicadas a la muestra de investigación, estas se encuentran procesadas de acuerdo a las variables estudiadas.

\section{Correlación de la hipótesis general}

H1: La gestión y políticas públicas impacta positivamente en el desarrollo de proyectos de inversión en el A.A.H.H. Olof Palme del distrito de Chilca, Cañete, 2021.

H0: La gestión y políticas públicas impacta negativamente en el desarrollo de proyectos de inversión en el A.A.H.H. Olof Palme del distrito de Chilca, Cañete, 2021. 
Tabla 1: Información del ajuste del modelo

\begin{tabular}{lcccccc}
\hline \multicolumn{1}{c}{ Modelo } & \multicolumn{2}{c}{ Criterio de ajuste del modelo } & \multicolumn{3}{c}{$\begin{array}{c}\text { Contrastes de la razón de } \\
\text { verosimilitud }\end{array}$} \\
\cline { 2 - 6 } & AIC & BIC & $\begin{array}{c}-2 \text { log } \\
\text { verosimilitud }\end{array}$ & $\begin{array}{c}\text { Chi- } \\
\text { cuadrado }\end{array}$ & Gl & Sig. \\
\hline Sólo la intersección & 687,976 & 781,282 & 613,976 & 358,741 & 74 &, 000 \\
Final & 477,234 & 757,153 & 255,234 & & & \\
\hline
\end{tabular}

Tabla 2: Pseudo R-cuadrado

\begin{tabular}{lc}
\hline Cox y Snell &, 980 \\
Nagelkerke &, 981 \\
McFadden &, 573 \\
\hline
\end{tabular}

Se observó de acuerdo a los resultados, el valor de Chi-cuadrado de 358,741 y el p-valor (valor de significancia) igual a ,000 frente a la significancia estadística de $\alpha$ igual a 0.05 (p-valor $<\alpha$ ), significando el rechazo de la hipótesis nula y aceptando la hipótesis propuesta por el investigador H1: "La gestión y políticas públicas impacta positivamente en el desarrollo de proyectos de inversión en el A.A.H.H. Olof Palme del distrito de Chilca, Cañete, 2021". Asimismo, se observa el valor del coeficiente Nagelkerke de $98,1 \%$, lo que también indica que es aceptada la hipótesis planteada por el investigador.

\section{Correlación de la Hipótesis específica 1}

H1: La gestión y políticas públicas impacta positivamente en el rol de los gobiernos regionales y locales para el desarrollo de proyectos de inversión en el A.A.H.H. Olof Palme del distrito de Chilca, Cañete, 2021.

H0: La gestión y políticas públicas impacta negativamente en el rol de los gobiernos regionales y locales en el desarrollo de proyectos de inversión en el A.A.H.H. Olof Palme del distrito de Chilca, Cañete, 2021.

Tabla 3: Información del ajuste del modelo

\begin{tabular}{lcccccc}
\hline \multicolumn{1}{c}{ Modelo } & \multicolumn{3}{c}{ Criterio de ajuste del modelo } & \multicolumn{2}{c}{$\begin{array}{c}\text { Contrastes de la razón de } \\
\text { verosimilitud }\end{array}$} \\
\hline & AIC & BIC & $\begin{array}{c}-2 \log \\
\text { verosimilitud }\end{array}$ & Chi-cuadrado & Gl & Sig. \\
& & & & & & \\
\hline Sólo la intersección & 521,335 & 571,771 & 481,335 & 210,371 & 40 &, 000 \\
Final & 390,964 & 542,272 & 270,964 & & & \\
\hline
\end{tabular}


Tabla 4: Pseudo R-cuadrado

\begin{tabular}{lc}
\hline Cox y Snell &, 898 \\
Nagelkerke &, 903 \\
McFadden &, 427 \\
\hline
\end{tabular}

Se observó de acuerdo a los resultados, el valor de Chi-cuadrado de 210,371 y el p-valor (valor de significancia) igual a ,000 frente a la significancia estadística de $\alpha$ igual a 0.05 ( $\mathrm{p}$-valor $<\alpha$ ), significando que se rechaza de la hipótesis nula y se acepta la hipótesis propuesta por el investigador H1: "La gestión y políticas públicas impacta positivamente en el rol de los gobiernos regionales y locales para el desarrollo de proyectos de inversión en el A.A.H.H. Olof Palme del distrito de Chilca, Cañete, 2021”. Asimismo, se evidencia la prueba de Pseudo R2, en la cual presenta al coeficiente de Nagelkerke con un valor de 90,3\%, lo que también indica que es aceptada la hipótesis planteada por el investigador.

\section{Correlación de la Hipótesis específica 2}

H1: La gestión y políticas públicas impacta positivamente en la inversión y crecimiento económico de los proyectos de inversión en el A.A.H.H. Olof Palme del distrito de Chilca, Cañete, 2021.

H0: La gestión y políticas públicas impacta negativamente en la inversión y crecimiento económico de los proyectos de inversión en el A.A.H.H. Olof Palme del distrito de Chilca, Cañete, 2021.

Tabla 5: Información del ajuste del modelo

\begin{tabular}{lcccccc}
\hline \multicolumn{1}{c}{ Modelo } & \multicolumn{3}{c}{ Criterio de ajuste del modelo } & \multicolumn{2}{c}{$\begin{array}{c}\text { Contrastes de la razón de } \\
\text { verosimilitud }\end{array}$} \\
\cline { 2 - 7 } & AIC & BIC & $\begin{array}{c}-2 \text { log } \\
\text { verosimilitud }\end{array}$ & Chi-cuadrado & Gl & Sig. \\
& & & & & \\
\hline Sólo la intersección & 463,269 & 498,574 & 435,269 & 170,809 & 28 &, 000 \\
Final & 348,460 & 454,375 & 264,460 & & & \\
\hline
\end{tabular}

Tabla 6: Pseudo R-cuadrado

\begin{tabular}{lc}
\hline Cox y Snell &, 844 \\
Nagelkerke &, 851 \\
McFadden &, 384 \\
\hline
\end{tabular}


Se observó de acuerdo a los resultados, el valor de Chi-cuadrado de 170,809 y el p-valor (valor de significancia) igual a ,000 frente a la significancia estadística de $\alpha$ igual a 0.05 ( $p$-valor $<\alpha$ ), significando que se rechaza de la hipótesis nula y se acepta la hipótesis propuesta por el investigador H1: "La gestión y políticas públicas impacta positivamente en la inversión y crecimiento económico de los proyectos de inversión en el A.A.H.H. Olof Palme del distrito de Chilca, Cañete, 2021". Asimismo, se evidencia la prueba de Pseudo R2, en la cual presenta al coeficiente de Nagelkerke con un valor de $85,1 \%$, lo que también indica que es aceptada la hipótesis planteada por el investigador.

\section{Correlación de la Hipótesis específica 3}

H1: La gestión y políticas públicas impacta positivamente en los lineamientos para la eficacia de los proyectos de inversión en el A.A.H.H. Olof Palme del distrito de Chilca, Cañete, 2021.

H0: La gestión y políticas públicas impacta negativamente en los lineamientos para la eficacia de los proyectos de inversión en el A.A.H.H. Olof Palme del distrito de Chilca, Cañete, 2021.

Tabla 7: Información del ajuste del modelo

\begin{tabular}{lcccccc}
\hline \multicolumn{1}{c}{ Modelo } & \multicolumn{3}{c}{ Criterio de ajuste del modelo } & \multicolumn{3}{c}{$\begin{array}{c}\text { Contrastes de la razón de } \\
\text { verosimilitud }\end{array}$} \\
\hline & AIC & BIC & $\begin{array}{c}-2 \text { log } \\
\text { verosimilitud }\end{array}$ & Chi-cuadrado & Gl & Sig. \\
\hline Sólo la intersección & 576,702 & 637,225 & 528,702 & 247,080 & 48 &, 000 \\
Final & 425,622 & 607,191 & 281,622 & & & \\
\hline
\end{tabular}

Tabla 8: Pseudo R-cuadrado

\begin{tabular}{lc}
\hline Cox y Snell &, 932 \\
Nagelkerke &, 934 \\
McFadden &, 458 \\
\hline
\end{tabular}

Se observó de acuerdo a los resultados, el valor de Chi-cuadrado de 247,080 y el p-valor (valor de significancia) igual a ,000 frente a la significancia estadística de $\alpha$ igual a 0.05 ( $\mathrm{p}$-valor $<\alpha$ ), significando que se rechaza de la hipótesis nula y se acepta la hipótesis propuesta por el investigador H1: "La gestión y políticas públicas impacta positivamente en los lineamientos para la eficacia de los proyectos de inversión en el A.A.H.H. Olof Palme del distrito de Chilca, Cañete, 2021”. Asimismo, se evidencia la prueba de Pseudo 
R2, en la cual presenta al coeficiente de Nagelkerke con un valor de 93,4\%, lo que también indica que es aceptada la hipótesis planteada por el investigador.

\section{Correlación de la Hipótesis específica 4}

H1: La gestión y políticas públicas impacta positivamente en las funciones del gobierno regional y local para el desarrollo de proyectos de inversión en el A.A.H.H. Olof Palme del distrito de Chilca, Cañete, 2021.

H0: La gestión y políticas públicas impacta negativamente en las funciones del gobierno regional y local para el desarrollo de proyectos de inversión en el A.A.H.H. Olof Palme del distrito de Chilca, Cañete, 2021.

Tabla 9: Información del ajuste del modelo

\begin{tabular}{lcccccc}
\hline \multicolumn{1}{c}{ Modelo } & \multicolumn{3}{c}{ Criterio de ajuste del modelo } & \multicolumn{3}{c}{$\begin{array}{c}\text { Contrastes de la razón } \\
\text { de verosimilitud }\end{array}$} \\
\cline { 2 - 7 } & AIC & BIC & $\begin{array}{c}-2 \text { log } \\
\text { verosimilitud }\end{array}$ & $\begin{array}{c}\text { Chi- } \\
\text { cuadrado }\end{array}$ & Gl & Sig. \\
\hline Sólo la intersección & 455,728 & 491,033 & 427,728 & 184,536 & 28 &, 000 \\
Final & 327,193 & 433,108 & 243,193 & & & \\
\hline
\end{tabular}

Tabla 10: Pseudo R-cuadrado

\begin{tabular}{ll}
\hline Cox y Snell &, 865 \\
Nagelkerke &, 873 \\
McFadden &, 421 \\
\hline
\end{tabular}

Se observó de acuerdo a los resultados, el valor de Chi-cuadrado de 184,536 y el p-valor (valor de significancia) igual a ,000 frente a la significancia estadística de $\alpha$ igual a 0.05 ( $\mathrm{p}$-valor $<\alpha$ ), significando que se rechaza de la hipótesis nula y se acepta la hipótesis propuesta por el investigador H1: "La gestión y políticas públicas impacta positivamente en las funciones del gobierno regional y local para el desarrollo de los proyectos de inversión en el A.A.H.H. Olof Palme del distrito de Chilca, Cañete, 2021”. Asimismo, se evidencia la prueba de Pseudo R2, en la cual presenta al coeficiente de Nagelkerke con un valor de $87,3 \%$, lo que también indica que es aceptada la hipótesis planteada por el investigador. 
Impacto de la gestión...

\section{B. Discusión}

En este punto la discusión se centra de dichos resultados encontrados a partir de los objetivos planteados, con apoyo de los antecedentes y teorías encontradas, describiendo los fenómenos para dar respuesta a la problemática planteada.

La gestión pública en el desarrollo de los proyectos de inversión, como se refleja en el estudio de Awaah et.al (2021), este señala que se desempeña un rol importante para garantizar la calidad de los recursos humanos en áreas como el sector salud, educación; ya que son amplios y aún se encuentran en la lucha contra la corrupción del gobierno. Contrastando con la presente investigación donde coeficiente Nagelkerke este presentó un valor de 98,1\% por lo cual se aceptó la hipótesis propuesta, llegando a la conclusión de que la gestión y políticas públicas impacta positivamente en el desarrollo de proyectos de inversión.

Del mismo modo, la gestión y políticas públicas impacta positivamente en el rol de los gobiernos regionales y locales para el desarrollo de proyectos de inversión, teniendo resultados positivos, tal como señalan Keers \& Fenema (2018) en su investigación; la gestión se integra a través de la participación y de las capacidades del personal, para direccionar el proyecto así como cumplir con las responsabilidades con el fin de obtener resultados óptimos.

La gestión y políticas públicas impacta positivamente en la inversión y crecimiento económico de los proyectos de inversión, lo cual se asemeja a lo que se menciona en la investigación de Algotson \& Svensson (2021), ya que se centra en el desarrollo que tienen los profesionales en el ámbito local y el crecimiento que tienen en el que incluyeron orientaciones variadas y estrategias para mejorar en distintos aspectos del crecimiento económico para aportar en la administración pública.

Además, la gestión y políticas públicas impacta positivamente en los lineamientos para la eficacia de los proyectos de inversión, se vincula con la investigación de Mueller (2020) señala que el sistema de las políticas públicas es complejo, sin embargo, este se basa en el control ya que compara los costes y beneficios con el objetivo de buscar alternatuvas adecuadas para la solución de los servicios que los ciudadanos requieren.

Finalmente, la gestión y políticas públicas impacta positivamente en las funciones del gobierno regional y local para el desarrollo de los proyectos de inversión, lo cual también se menciona en la investigación de Holst (2018) donde existen factores que son 
importantes para poder cumplir con lo que el gobierno plantea y quiere reforzar, dado que en ellos se depositó la confianza de las personas porque ellos son los más adecuados para tomar las decisiones que favorezcan al desarrollo del país.

\section{CONCLUSIÓN O CONSIDERACIONES FINALES}

\section{Conclusiones}

- Como primera conclusión, se mostró que a través del coeficiente Nagelkerke se obtuvo un valor de 98,1\% por lo cual se aceptó la hipótesis propuesta, llegando a la conclusión de que la gestión y políticas públicas impacta positivamente en el desarrollo de proyectos de inversión en el A.A.H.H. Olof Palme del distrito de Chilca, Cañete, 2021.

- Como segunda conclusión, a través del coeficiente Nagelkerke se puede concluir que la gestión y políticas públicas impacta positivamente en el rol de los gobiernos regionales y locales para el desarrollo de proyectos de inversión en el A.A.H.H. Olof Palme del distrito de Chilca, Cañete, 2021, esto debido a que los resultados muestran un $90,3 \%$ lo que significa que la hipótesis planteada fue aprobada.

- Como tercera conclusión, se pudo identificar que la gestión y políticas públicas impacta positivamente en la inversión y crecimiento económico de los proyectos de inversión en el A.A.H.H. Olof Palme del distrito de Chilca, Cañete, 2021 a través del coeficiente Nagelkerke, donde se evidenció un valor de 85,1\% aceptando así la hipótesis planteada por el investigador.

- Como cuarta conclusión mediante el uso del coeficiente Nagelkerke se pudo determinar que la gestión y políticas públicas impacta positivamente en los lineamientos para la eficacia de los proyectos de inversión en el A.A.H.H. Olof Palme del distrito de Chilca, Cañete, 2021 ya que presentó un valor de 93,4\%, lo que indica que es aceptada la hipótesis planteada.

- Finalmente, se concluye que la gestión y políticas públicas impacta positivamente en las funciones del gobierno regional y local para el desarrollo de los proyectos de inversión en el A.A.H.H. Olof Palme del distrito de Chilca, Cañete, 2021 ya que por medio del coeficiente Nagelkerke se pudo aceptar la hipótesis planteada por el investigador debido a que se evidenció un valor de $87,3 \%$. 
Impacto de la gestión...

\section{Recomendaciones}

- Se recomienda contar con estrategias que permitan la orientación en cuanto a la gestión y políticas públicas beneficiosas que se pretendan desarrollar mediante proyectos de inversión en el A.A.H.H. Olof Palme.

- Se recomienda a los gobiernos regionales y locales fiscalizar el desarrollo de proyectos que se lleven a cabo en su circunscripción territorial, ello con la finalidad de procurar y velar por el beneficio de la ciudadanía.

- Se recomienda la formulación de medidas, planes y políticas orientadas a promover la inversión pública y privada, a fin de lograr un mayor crecimiento económico y calidad de vida a la población.

- Se recomienda contar con un plan periódico que tenga como fin supervisar y realizar un seguimiento a los proyectos que se lleven a cabo en las diferentes circunscripciones, ello a fin de poder evidenciar posibles fallas y tomar medidas de manera oportuna.

- Se recomienda brindar capacitaciones al personal que realizará la fiscalización y seguimiento de este tipo de proyectos de inversión, ello a fin de evitar posibles errores.

\section{LISTA DE REFERENCIAS}

Algotson, A., \& Svensson, P. (2021). Conceptualizing local development practitioners: creators, coordinators or inside lobbyists? Urban Governance. https://www.sciencedirect.com/science/article/pii/S266432862100005X

Arundel, A., Bloch, C., \& Ferguson, B. (2019). Advancing innovation in the public sector: Aligning innovation measurement with policy goals. Research Policy, 48(3), 789798. doi:https://doi.org/10.1016/j.respol.2018.12.001

Avalos, K., \& Brenes, P. (2021). The impact of covid-19 in higher education classes, in the english teaching for I and II cycle mayor at Universidad Hispanoamericana. Ciencia Latina Revista Científica Multidisciplinar, 5(5). doi:https://doi.org/10.37811/cl_rcm.v5i5.919

Awaah, F., Okebukola, P., Alpha Alfa, A., Yeboah, S., Anagba, K., \& Arkorful, H. (2021). Developing public administration education by focusing on difficult key concepts: The case of Nigeria and Ghana. Social Sciences \& Humanities Open. https://www.sciencedirect.com/science/article/pii/S2590291121000322 
Brunet, M. (2019). Governance-as-practice for major public infrastructure projects: A case of multilevel project governing. International Journal of Project Management, 37(2), 283-297. doi:https://doi.org/10.1016/j.ijproman.2018.02.007 da Assunção Moutinho, J., \& Rabechini Junior, R. (2020). Project management in the public context: research field mapping. Revista de Administração Pública. https://www.scielo.br/j/rap/a/mPhSXgwMnT9cgrQgFb5gBnJ/?lang=en

Damascene Twizeyimana, J., \& Andersson, A. (2019). The public value of EGovernment - A literature review. Government Information Quarterly, 36(2), 167-178. doi:https://doi.org/10.1016/j.giq.2019.01.001

Dauzón, L., \& Izquierdo, M. (2020). Digital natives and technology for L2 learning outside of the classroom. Apertura, 12(1), 72-87. doi:https://doi.org/10.32870/ap.vl2nl.1801

Fleury Teixeira, S. M., Subirats, J., Lacerda, D., \& Lacerda, I. (2018). Public policies and the city: producing inclusive urban spaces. Revista de Administração Pública, 52(6). doi:https://doi.org/10.1590/0034-761220180306

Gutiérrez Ossa, J. A., Restrepo Avendaño, R. D., \& Zapata Hoyos, J. S. (2017). Formulación, implementación y evaluación de políticas públicas desde los enfoques, fines y funciones del Estado. CES Derecho. http://www.scielo.org.co/pdf/cesd/v8n2/v8n2a08.pdf

Hansonn, L. (2020). Public administrators' roles in the policy adaptation of transport directives: How knowledge is created and reproduced. Transport Policy, 98, 208216. doi:https://doi.org/10.1016/j.tranpol.2019.10.008

Holst Volden, G. (2018). Public project success as seen in a broad perspective.: Lessons from a meta-evaluation of 20 infrastructure projects in Norway. Science Direct. https://www.sciencedirect.com/science/article/pii/S0149718917303208

Hong, S., \& Ryu, J. (2019). Crowdfunding public projects: Collaborative governance for achieving citizen co-funding of public goods. Government Information Quarterly, 36(1), 145-153. doi:https://doi.org/10.1016/j.giq.2018.11.009

Huacchillo Pardo, L. A., Torres Surita, N., \& Ramos Farroñan, E. V. (2020). Contributing factor for Growth and Business Entrepreneurship. Public Investment. http://scielo.sld.cu/pdf/rus/v12n2/2218-3620-rus-12-02-350.pdf 
Jiang, S. (2021). The relationship between Public and Private Health Investment and economic development based on the Overlapping Generationes (OLG) Model. Revista Brasileira de Medicina do Esporte. https://www.scielo.br/j/rbme/a/wWnR36NKDqDnKcBvV93W7fF/

Keers, B., \& Fenema, P. (2018). Managing risks in public-private partnership formation projects. International Journal of Project Management. https://www.sciencedirect.com/science/article/abs/pii/S0263786317309092

Lakerveld, J., Woods , C., Hebestreit, A., Brenner, H., Flechtner, M., Harrington, J., . . Ahrens, W. (2020). Advancing the evidence base for public policies impacting on dietary behaviour, physical activity and sedentary behaviour in Europe: The Policy Evaluation Network promoting a multidisciplinary approach. Food Policy, 96. doi:https://doi.org/10.1016/j.foodpol.2020.101873

Morveli Salas, G. (2021). Enfoques de la Gestión pública y su influencia en el gobierno peruano 1990 al 2020. Ciencia Latina Revista Multidisciplinar. https://ciencialatina.org/index.php/cienciala/article/view/546

Mueller, B. (2020). Why public policies fail: Policymaking under complexity. EconomiA. https://www.sciencedirect.com/science/article/pii/S1517758019300931

Ola-Awo, W. A., Alayande, A., Olarewaju Bashir, G., \& Oyekunle Oyewobi, L. (2021). Critical success factors for effective internal construction stakeholder management in Nigeria. Acta Structilia. http://www.scielo.org.za/scielo.php?script=sci_arttext\&pid=S241504872021000100001\&lng=en\&nrm=iso\&tlng=en

Soto Arévalo, R. (2021). Eficiencia en la ejecución de proyectos de inversión. Ciencia Latina Revista

Multidisciplinar. https://ciencialatina.org/index.php/cienciala/article/view/378

Wagner, R., Huemann, M., \& Radujkovic, M. (2021). The influence of project management associations on projectification of society - An institutional $\begin{array}{llll}\text { perspective. Project Leadership and } & \text { Society, } 2 .\end{array}$ doi:https://doi.org/10.1016/j.plas.2021.100021

Wilson, C. (2022). Public engagement and AI: A values analysis of national strategies. Government Information Quarterly, 39(1). doi:https://doi.org/10.1016/j.giq.2021.101652 
Zavaleta, J., \& Cruz, M. (2020). Productivity and effective demand: assessing the disaggregate public spending. Investigación económica. http://www.scielo.org.mx/scielo.php?script=sci_arttext\&pid=S018516672019000300107 